hep-th/0603188

\title{
Wilson-'t Hooft operators and the theta angle
}

\author{
Måns Henningson \\ Department of Fundamental Physics \\ Chalmers University of Technology \\ S-412 96 Göteborg, Sweden
}

\begin{abstract}
:
We consider $(3+1)$-dimensional $S U(N) / \mathbb{Z}_{N}$ Yang-Mills theory on a space-time with a compact spatial direction, and prove the following result: Under a continuous increase of the theta angle $\theta \rightarrow \theta+2 \pi$, a 't Hooft operator $T(\gamma)$ associated with a closed spatial curve $\gamma$ that winds around the compact direction undergoes a monodromy $T(\gamma) \rightarrow T^{\prime}(\gamma)$. The new 't Hooft operator $T^{\prime}(\gamma)$ transforms under large gauge transformations in the same way as the product $T(\gamma) W(\gamma)$, where $W(\gamma)$ is the Wilson operator associated with the curve $\gamma$ and the fundamental representation of $S U(N)$.
\end{abstract}




\section{Introduction}

Wilson operators and 't Hooft operators constitute important observables in non-abelian Yang-Mills theory in $d=3+1$ dimensions. In this paper, we will consider the gauge group

$$
G \simeq S U(N) / C,
$$

where $C \simeq \mathbb{Z}_{N}$ denotes the center of $S U(N)$. The basic Wilson operator $W(\gamma)$ associated with a closed spatial curve $\gamma$ is then defined as

$$
W(\gamma)=\frac{1}{N} \operatorname{Tr}\left(P \exp \int_{\gamma} A\right),
$$

where $A$ is the connection one-form, $P$ denotes path ordering along $\gamma$, and $\operatorname{Tr}$ is the trace in the fundamental representation of $S U(N)$. The operator $W(\gamma)$ is invariant under gauge transformations that can be continuosly deformed to the identity transformation. Under a general gauge transformation, $W(\gamma)$ is multiplied by an $N$-th root of unity determined by the class in $\pi_{1}(G) \simeq \mathbb{Z}_{N}$ of (the restriction to $\gamma$ of) the gauge transformation.

The definition of the corresponding 't Hooft operator $T(\gamma)$ is less explicit [1]: On the complement of $\gamma$ in space, $T(\gamma)$ is given by a $G$ valued gauge transformation, whose restriction to another closed curve that links $\gamma$ once represents the image of the generator 1 of $\mathbb{Z}_{N}$ under the isomorphism $\mathbb{Z}_{N} \simeq \pi_{1}(G)$. Such a transformation has a well-defined action on all the fields of the theory, but is obviously singular at the locus of $\gamma$. By deforming the transformation over a tubular neighbourhood of $\gamma$, we regularize it to a smooth transformation defined over all of space-time. The precise form of the regularization is of no consequence for the arguments of the present paper; the important point is that the resulting field configuration is smooth everywhere. The regularized transformation is however not a gauge transformation, so the 't Hooft operator $T(\gamma)$ thus defined has a non-trivial action also on gauge invariant states.

This definition of the 't Hooft operator in terms of a singular gauge transformation is ambigious in the sense that it allows for the multiplication of $T(\gamma)$ by a phase-factor, which may be an arbitrary gauge-invariant functional of the fields of the theory. It may even be impossible to give a globally valid prescription for fixing this ambiguity. Indeed, it has been stated in several papers (see for example [2] 3] 4), that under a smooth increase $\theta \rightarrow \theta+2 \pi$ of the theta angle, $T(\gamma)$ undergoes a monodromy

$$
T(\gamma) \rightarrow T^{\prime}(\gamma),
$$

where the new 't Hooft operator $T^{\prime}(\gamma)$ behaves as the product $T(\gamma) W(\gamma)$; it could be called a Wilson-'t Hooft operator. (On the other hand, the explicit expression (2) shows that the corresponding monodromy of the Wilson operator $W(\gamma)$ is trivial; $W(\gamma) \rightarrow W(\gamma)$.) This monodromy of operators associated with closed spatial curves is analogous to the Witten effect [5], which amounts to an increase of the electric charge of a magnetically charged dyonic particle state as $\theta \rightarrow \theta+2 \pi$ continuously. 
However, we are not aware of any published proof of the monodromy transformation (3). The aim of the present paper is to provide such a proof, based on a topological obstruction that prevents a global definition of $T(\gamma)$. The obstruction will only be present if the curve $\gamma$ represents a non-trivial homotopy class. We will therefore consider the theory on a spatial three-manifold $X$ of the form

$$
X \simeq S^{1} \times \mathbb{R}^{2},
$$

and let $\gamma$ wind once around the $S^{1}$ factor of $X$. However, even if there is no topological obstruction against a global definition of the 't Hooft operator if we take the spatial manifold as $\mathbb{R}^{3}$, it is certainly natural to assume a similar monodromy transformation also in this case.

In the next section, we will review the interpretation of the 't Hooft operator $T(\gamma)$ in terms of the topology of principal $G$ bundles over space. In section three, we will consider the topology of the group of gauge transformations. A gauge transformation may be winded in two different ways: along the curve $\gamma$, or over three-space as a whole. As discussed above, gauge transformations that are winded along $\gamma$ have a non-trivial action on the Wilson operator. The transformation properties under gauge transformations that are winded over three-space as a whole are described by the theta angle. In section four, we will show how the interplay of these effects leads to the monodromy property (3).

\section{2 't Hooft operators}

We begin by reviewing the classification of principal $G \simeq S U(N) / C$ bundles over a low-dimensional compact connected space $B$. This follows from the first few homotopy groups of $G$ :

$$
\pi_{i}(G) \simeq \begin{cases}0, & i=0 \\ \mathbb{Z}_{N}, & i=1 \\ 0, & i=2 \\ \mathbb{Z}, & i=3\end{cases}
$$

Thus, for a one-dimensional base space $B$, all $G$ bundles are trivial. For a twoor three-dimensional $B$, they are classified by a characteristic class

$$
w_{2} \in H^{2}\left(B, \mathbb{Z}_{N}\right),
$$

known as the second Stiefel-Whitney class in mathematics or the discrete magnetic flux in physics. For a four-dimensional $B$ there is an additional characteristic class

$$
c_{2} \in H^{4}(B, \mathbb{Q}),
$$

known as the second Chern class or the instanton number. It is related to the second Stiefel-Whitney class $w_{2}$ as

$$
c_{2}=\frac{1}{2}\left(\frac{1}{N}-1\right) \bar{w}_{2} \cup \bar{w}_{2} \bmod H^{4}(B, \mathbb{Z}),
$$


where $\bar{w}_{2} \in H^{2}(B, \mathbb{Z})$ denotes an arbitrary lifting of $w_{2}$ to an integral class. (An instructive proof of this relation can be found in e.g. [6].) In higher dimensions, there are further invariants, but they will not be needed in the present paper.

Consider now a state of finite energy in Yang-Mills theory with gauge group $G$ on the spatial manifold $X \simeq S^{1} \times \mathbb{R}^{2}$. As we go to infinity in the $\mathbb{R}^{2}$ factor, all physical data must approach their vacuum values. We may therefore add the points at infinity, thereby replacing $X$ by the compact space

$$
X^{\prime} \simeq S^{1} \times S^{2} .
$$

However, while a $G$ bundle $P$ over $X$ is necessarily trivial $\left(\right.$ since $H^{2}\left(X, \mathbb{Z}_{N}\right) \simeq$ 0 ), this is not so for a $G$ bundle $P^{\prime}$ over $X^{\prime}$; according to the previous paragraph, such bundles are classified by a characteristic class $w_{2}^{\prime} \in H^{2}\left(X^{\prime}, \mathbb{Z}_{N}\right) \simeq \mathbb{Z}_{N}$.

It is now easy to understand the action of an 't Hooft operator $T(\gamma)$ associated with a closed curve $\gamma$ that winds once around the $S^{1}$ factor of $X$ or $X^{\prime}$ : When acting on a state $|\psi\rangle$ with a definite value $w_{2}^{\prime}$ of the second StiefelWhitney class, it produces another state $|\tilde{\psi}>=T(\gamma)| \psi\rangle$ for which the second Stiefel-Whitney class takes the value $\tilde{w}_{2}^{\prime}$ given by

$$
\tilde{w}_{2}^{\prime}=w_{2}^{\prime}+1
$$

(In this formula, we identify a class in $H^{2}\left(X^{\prime}, \mathbb{Z}_{N}\right)$ with its image under the isomorphism $H^{2}\left(X^{\prime}, \mathbb{Z}_{N}\right) \simeq \mathbb{Z}_{N}$. $)$

\section{$3 \quad$ Large gauge transformations}

This section is largely inspired by [7].

Let $P^{\prime}$ be a $G$ bundle over $X^{\prime} \simeq S^{1} \times S^{2}$, characterized by its value $w_{2}^{\prime} \in$ $H^{2}\left(X^{\prime}, \mathbb{Z}_{N}\right)$ of the second Stiefel-Whitney class, as described in the previous section. We let $\mathcal{G}$ denote the group of gauge transformations, i.e. the group of bundle automorphisms of $P^{\prime}$. It is not connected; the component of $\mathcal{G}$ containing the identity transformation is a normal subgroup, which we denote as $\mathcal{G}_{0}$. Physical states must be invariant under $\mathcal{G}_{0}$, but they need not be invariant under all of $\mathcal{G}$. Their transformation properties may be given by an arbitrary character of the quotient group of homotopy classes of gauge transformations

$$
\Lambda \simeq \mathcal{G} / \mathcal{G}_{0} .
$$

We will need to understand the structure of the discrete abelian group $\Lambda$. Let $\Lambda_{\gamma} \simeq \pi_{1}(G) \simeq \mathbb{Z}_{N}$ be the group of homotopy classes of gauge transformations for the trivial bundle over the spatial curve $\gamma$ that is obtained by restricting the bundle $P^{\prime}$ to $\gamma$. Let $\Lambda_{0} \simeq \pi_{3}(G) \simeq \mathbb{Z}$ be the subgroup of $\Lambda$ consisting of homotopy classes of gauge transformations of $P^{\prime}$ that are trivial when restricted to $\gamma$. We thus have a short exact sequence

$$
0 \rightarrow \Lambda_{0} \stackrel{i}{\rightarrow} \Lambda \stackrel{r}{\rightarrow} \Lambda_{\gamma} \rightarrow 0
$$


where $i$ and $r$ are the obvious inclusion and restriction maps respectively. In other words, the group $\Lambda$ is an extension of $\Lambda_{\gamma} \simeq \mathbb{Z}_{N}$ by $\Lambda_{0} \simeq \mathbb{Z}$. To describe this extension precisely, we choose a $\lambda \in \Lambda$ such that $r(\lambda)$ equals the image of the generator 1 of $\mathbb{Z}_{N}$ under the isomorphism $\mathbb{Z}_{N} \simeq \Lambda_{\gamma}$. Since $\lambda^{N} \in \operatorname{ker} r$ and the sequence is exact, $\lambda^{N} \in \operatorname{Im} i$, so

$$
\lambda^{N}=\Omega^{k},
$$

where $\Omega$ is the generator of $\Lambda_{0} \simeq \mathbb{Z}$ and $k$ is some integer, which depends on the choice of $\lambda$. (Here we have switched to a multiplicative rather than additive notation for the group operations.)

To compute the integer $k$, we consider the four-dimensional space

$$
Y \simeq S^{1} \times X^{\prime} \simeq S^{1} \times S^{1} \times S^{2} .
$$

(As will become clear, this auxiliary space should not be thought of as a spacetime.) We construct two $G$ bundles $P^{\lambda}$ and $P^{\Omega}$ over $Y$ by first extending the given bundle $P$ over the cylinder $I \times X^{\prime}$, where $I$ is an interval, and then gluing the ends together with gluing data $\lambda$ or $\Omega$ respectively. We then have that

$$
N c_{2}^{\lambda}=k c_{2}^{\Omega},
$$

where $c_{2}^{\lambda}$ and $c_{2}^{\Omega}$ are the second Chern classes of the bundles $P^{\lambda}$ and $P^{\Omega}$ respectively. The class $c_{2}^{\Omega} \in H^{4}(Y, \mathbb{Q})$ is given by the image of the element 1 of $\mathbb{Q}$ under the isomorphism $\mathbb{Q} \simeq H^{4}(Y, \mathbb{Q})$. According to (8), the class $c_{2}^{\lambda} \in H^{4}(Y, \mathbb{Q})$ is determined modulo $H^{4}(Y, \mathbb{Z})$ by the second Stiefel-Whitney class $w_{2}^{\lambda} \in H^{2}\left(Y, \mathbb{Z}_{N}\right)$ of $P^{\lambda}$. The latter class is determined by its restrictions to the factors $S^{1} \times S^{1}$ and $S^{2}$ on the right hand side of (14). The restriction of $w_{2}^{\lambda}$ to $S^{1} \times S^{1}$ is in fact given by $r(\lambda) \in H^{2}\left(S^{1} \times S^{1}, \mathbb{Z}_{N}\right) \simeq \Lambda_{\gamma}$. The restriction of $w_{2}^{\lambda}$ to $S^{2}$ equals the second Stiefel-Whitney class $w_{2}^{\prime} \in H^{2}\left(S^{2}, \mathbb{Z}_{N}\right) \simeq H^{2}\left(X^{\prime}, \mathbb{Z}_{N}\right)$ of $P^{\prime}$. Thus

$$
w_{2}^{\lambda}=p_{1}^{*}(r(\lambda))+p_{2}^{*}\left(w_{2}^{\prime}\right),
$$

where $p_{1}$ and $p_{2}$ are the projections from $Y$ to $S^{1} \times S^{1}$ and $S^{2}$ respectively. A small calculation now gives

$$
c_{2}^{\lambda}=\frac{1}{N} p_{1}^{*}(r(\lambda)) \cup p_{2}^{*}\left(w_{2}^{\prime}\right) \bmod H^{4}(Y, \mathbb{Z}) .
$$

Putting everything together, we find that $k=w_{2}^{\prime} \bmod N$, where $w_{2}^{\prime}$ denotes the image of the second Stiefel-Whitney class of $P^{\prime}$ under the isomorphism $H^{2}\left(X^{\prime}, \mathbb{Z}_{N}\right) \simeq \mathbb{Z}_{N}$. (Since $\lambda$ is only defined modulo $\Omega$, we can only determine $k$ modulo $N$.)

In summary, we have found that the group $\Lambda$ is generated by the elements $\lambda$ and $\Omega$, subject to the relation

$$
\lambda^{N}=\Omega^{w_{2}^{\prime} \bmod N} .
$$




\section{The monodromy}

A physical state $|\psi\rangle$ is characterized by a certain value $w_{2}^{\prime}$ of the second StiefelWhitney class, as described in section two. Its transformation properties under the discrete abelian group $\Lambda$ described in the previous section can be specified by the eigenvalues $e^{i \theta}$ and $e^{i \phi}$ of the generators $\Omega$ and $\lambda$ respectively:

$$
\begin{aligned}
\Omega|\psi\rangle & =e^{i \theta}|\psi\rangle \\
\lambda|\psi\rangle & =e^{i \phi}|\psi\rangle .
\end{aligned}
$$

As the notation suggests, $\theta$ is indeed the theta angle parameter of Yang-Mills theory. The relation (18) implies that

$$
e^{i \phi N}=e^{i\left(w_{2}^{\prime}+n N\right) \theta}
$$

for some integer $n$. If we follow a particular solution to this equation under a continuous increase $\theta \rightarrow \theta+2 \pi$, the eigenvalue $e^{i \phi}$ undergoes the monodromy

$$
e^{i \phi} \rightarrow e^{i \phi} e^{2 \pi i w_{2}^{\prime} / N}
$$

Acting with an 't Hooft operator $T(\gamma)$ on $|\psi\rangle$ produces another state $|\tilde{\psi}\rangle=$ $T(\gamma)|\psi\rangle$ with the value $\tilde{w}_{2}^{\prime}=w_{2}^{\prime}+1$ of the second Stiefel-Whitney class. Repeating the above argument, we find that the corresponding eigenvalue $e^{i \tilde{\phi}}$ of the generator $\lambda$ undergoes the monodromy

$$
e^{i \tilde{\phi}} \rightarrow e^{i \tilde{\phi}} e^{2 \pi i\left(w_{2}^{\prime}+1\right) / N} .
$$

The different monodromy properties of the two states mean that the 't Hooft operator must undergo a monodromy

$$
T(\gamma) \rightarrow T^{\prime}(\gamma)
$$

The quotient $\hat{W}(\gamma)=T^{\prime}(\gamma) T^{-1}(\gamma)$ can be characterized by its transformation property under $\lambda$ :

$$
\lambda \hat{W}(\gamma) \lambda^{-1}=e^{2 \pi i / N} \hat{W}(\gamma) .
$$

But this agrees with the transformation property of the Wilson operator $W(\gamma)$ in the fundamental representation of $S U(N)$ as defined in (2). So although the present arguments do not give an exact description of $T^{\prime}(\gamma)$ (which would depend on the precise prescription for regularizing the 't Hooft operators in the vicinity of $\gamma$ ), we can conclude that $T^{\prime}(\gamma)$ indeed transforms in the same way as the product $T(\gamma) W(\gamma)$ under gauge transformations.

I am supported by a Research Fellowship from the Royal Swedish Academy of Sciences. 


\section{References}

[1] G. 't Hooft, "On The Phase Transition Towards Permanent Quark Confinement," Nucl. Phys. B 138 (1978) 1.

[2] M. Bianchi, M. B. Green and S. Kovacs, "Instanton corrections to circular Wilson loops in $\mathrm{N}=4$ supersymmetric Yang-Mills," JHEP 0204 (2002) 040 arXiv:hep-th/0202003.

[3] F. Cachazo, N. Seiberg and E. Witten, "Phases of $\mathrm{N}=1$ supersymmetric gauge theories and matrices," JHEP 0302 (2003) 042 arXiv:hep-th/0301006.

[4] A. Kapustin, "Wilson-'t Hooft operators in four-dimensional gauge theories and S-duality," arXiv:hep-th/0501015

[5] E. Witten, "Dyons Of Charge E Theta / 2 Pi," Phys. Lett. B 86, 283 (1979).

[6] C. Vafa and E. Witten, "A Strong coupling test of S duality," Nucl. Phys. B 431, 3 (1994) arXiv:hep-th/9408074.

[7] E. Witten, "Supersymmetric index in four-dimensional gauge theories," Adv. Theor. Math. Phys. 5 (2002) 841 arXiv:hep-th/0006010. 